\title{
VIOLENCIA OBSTÉTRICA: A PROPÓSITO DE UNA SERIE DE CASOS
}

\begin{abstract}
RESUMEN
Con el objetivo de visibilizar la probable ocurrencia de falta de respeto y/o maltrato durante el acceso a la atención del parto, se realizó la búsqueda de noticias relacionadas a la atención del parto, negación de la atención del parto o la atención no oportuna del parto durante el año 2017 en los motores de búsqueda

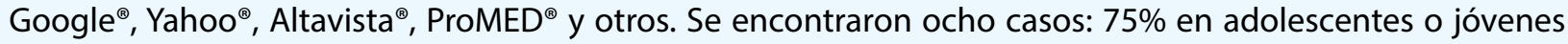
y tres de cada cuatro casos ocurrieron en Lima y Callao que evidenciarían deficiencias en el acceso a la atención del parto de calidad y podrían ser la punta del iceberg de una inadecuada y deshumanizada atención integral de la salud materna.
\end{abstract}

Palabras clave: Parto humanizado; Calidad de la atención de salud; Mujeres embarazadas. (fuente: DeCS BIREME)

\begin{abstract}
In order to make visible the probable occurrence of lack of respect and / or mistreatment during access to childbirth care, the search was made for news related to delivery care, denial of delivery care or no-timely care birth during 2017 in the search engines Google ${ }^{\oplus}, \mathrm{Yahoo}^{\oplus}$, Altavista $^{\oplus}, \mathrm{ProMED}^{\circledR}$ and others. Eight cases were found: $75 \%$ in adolescents or young people and three out of four cases occurred in Lima and Callao that would show deficiencies in access to quality birth care and could be the tip of the iceberg of inadequate and dehumanized comprehensive care maternal health
\end{abstract}

Key words: Obstetric delivery; Quality of health care; Pregnant women. (source: MeSH NLM)

\section{INTRODUCCIÓN}

El parto es un proceso normal y natural, un periodo vulnerable para la salud de la mujer, en el cual el entorno y las actuaciones sanitarias tienen una gran influencia' para evitar o reducir el riesgo morbilidad y mortalidad materna. Esto se logra cuando la gestante accede a servicios de salud de calidad con oportunidad, debido a que en ellos encuentra personal de salud que reconoce los signos de alarma, diagnostica y maneja las complicaciones que se presentan en forma apropiada y oportuna, respetando los derechos de la gestante y su recién nacido a una atención humanizada del parto².
El Perú es uno de los cinco países de América Latina y El Caribe que más ha avanzado en la reducción de la mortalidad materna ${ }^{3,4}$ y a esto se suma la mejora en las coberturas de parto institucional ${ }^{5}$; sin embargo, en los últimos meses del año 2017, diversos medios de comunicación difundieron eventos que reflejarían una atención no oportuna e inadecuada de las gestantes por parte del personal de salud, lo que motivó a elaborar el presente reporte que tiene como objetivo visibilizar la probable ocurrencia de falta de respeto y/o maltrato durante el acceso a la atención del parto.

\footnotetext{
'Natural and Social Sciences Research

2 Universidad Nacional Daniel Alcides Carrión

aédico cirujano

bicenciada en Comunicación Social

¿icenciada en Enfermería
}

Citar como: Alfredo Enrique Oyola-García, Ada Liz Palpán-Guerra, Melisa Pamela Quispe-Llanzo. Violencia obstétrica: A propósito de una serie de casos. [Artículo Original].2018;18(4):14-19. (Octubre 2018). DOI 10.25176/RFMH.v18.n4.1730 


\section{EL ESTUDIO}

Debido a que los medios de comunicación son una importante fuente de información de eventos de importancia para la salud pública ${ }^{6}$, se realizó la búsqueda de noticias relacionadas a la atención del parto, negación de la atención del parto o la atención no oportuna del parto durante el año 2017, mediante palabras clave y alertas en los motores de búsqueda $\left(\right.$ Google $^{\oplus}$, Yahoo ${ }^{\circledast}$, Altavista ${ }^{\oplus}$, ProMED $^{\circledast}$ y otros); teniendo en consideración dos definiciones: a) el "parto institucional" es aquel "ocurrido en un establecimiento de salud (hospital, centro o puesto de salud) que incluye el parto vaginal y abdominal (cesárea) ${ }^{\prime 2}$; y b) los casos en que se abandona o niega la atención o no se atiende oportuna y eficazmente las emergencias obstétricas se denomina "falta de respeto y maltrato durante la atención del parto" o "violencia obstétrica"7.

\section{HALLAZGOS}

Se encontraron ocho casos: $75 \%$ en adolescentes o jóvenes y tres de cada cuatro casos ocurrieron en Lima y Callao. Asimismo, a pesar de ser "partos institucionales" en su mayoría, no denotan la calidad ni la oportunidad que reviste este indicador pues más de la mitad de los partos se produjeron en los servicios higiénicos, a pesar que -en todos los casos- hubo solicitud y contacto previo con personal de salud del mismo establecimiento para dicha atención (Tabla 1).

Tabla 1. Casos de violencia obstétrica durante el acceso a la atención del parto reportados por medios de comunicación, 2017.

\begin{tabular}{|c|c|c|c|c|c|c|}
\hline $\mathbf{N}^{\circ}$ & FECHA & $\begin{array}{l}\text { ESTABLECIMIENTO } \\
\text { DE SALUD }\end{array}$ & $\begin{array}{l}\text { LUGAR DE } \\
\text { PARTO }\end{array}$ & EDAD & $\begin{array}{c}\text { MEDIO DE } \\
\text { COMUNICACIÓN }\end{array}$ & ENLACE \\
\hline 1 & $06 / 05 / 2017$ & $\begin{array}{l}\text { Hospital III de } \\
\text { EsSalud (Puno) }\end{array}$ & $\begin{array}{l}\text { Servicios } \\
\text { higiénicos }\end{array}$ & 25 años & Sin Fronteras & $\begin{array}{l}\text { http://www.diariosinfronteras. } \\
\text { pe/2017/05/08/indignante-madre- } \\
\text { da-a-luz-en-un-bano-de-essalud- } \\
\text { puno/ }\end{array}$ \\
\hline 2 & 29/05/2017 & $\begin{array}{l}\text { Hospital Sergio } \\
\text { Bernales (Lima) }\end{array}$ & $\begin{array}{l}\text { Servicios } \\
\text { higiénicos }\end{array}$ & 24 años & América tv & $\begin{array}{l}\text { http://www.americatv.com.pe/ } \\
\text { noticias/actualidad/mujer-tuvo- } \\
\text { que-dar-luz-bano-hospital-collique- } \\
\text { comas-n278615 }\end{array}$ \\
\hline 3 & $08 / 06 / 2017$ & $\begin{array}{l}\text { Hospital Hipólito } \\
\text { Unanue (Lima) }\end{array}$ & $\begin{array}{l}\text { Servicios } \\
\text { higiénicos }\end{array}$ & Adolescente & América tv & $\begin{array}{l}\text { http://www.americatv.com. } \\
\text { pe/noticias/actualidad/mujer- } \\
\text { dio-luz-bano-hospital-hipolito- } \\
\text { unanue-n279523 }\end{array}$ \\
\hline 4 & $21 / 06 / 2017$ & $\begin{array}{l}\text { Hospital Ramiro } \\
\text { Prialé (Junín) }\end{array}$ & Pasillo & 28 años & Sin Fronteras & $\begin{array}{l}\text { http://www.diariosinfronteras. } \\
\text { pe/2017/06/21/indignante-mujer- } \\
\text { da-a-luz-en-pasillo-de-hospital-en- } \\
\text { junin/ }\end{array}$ \\
\hline 5 & $19 / 07 / 2017$ & $\begin{array}{l}\text { Hospital de } \\
\text { Huaycán (Lima) }\end{array}$ & $\begin{array}{l}\text { Servicios } \\
\text { higiénicos }\end{array}$ & 14 años & Peru 21 & $\begin{array}{l}\text { http://peru21.pe/actualidad/ } \\
\text { huaycan-adolescente-14-anos-da- } \\
\text { luz-bano-hospital-2290050 }\end{array}$ \\
\hline 6 & 08/08/2017 & $\begin{array}{l}\text { Hospital Luis } \\
\text { Negreiros (Callao) }\end{array}$ & Pasillo & 37 años & Latina & $\begin{array}{l}\text { http://www.latina.pe/noticias/90/ } \\
\text { locales/callao-mujer-da-a-luz-en- } \\
\text { pasadizo-del-hospital-luis-negreiros }\end{array}$ \\
\hline 7 & $07 / 09 / 2017$ & $\begin{array}{l}\text { Hospital de Ate } \\
\text { Vitarte (Lima) }\end{array}$ & Patio & 26 años & Latina & $\begin{array}{l}\text { http://www.latina.pe/noticias/90/ } \\
\text { locales/denuncian-maltrato-a- } \\
\text { gestante-que-dio-a-luz-en-patio-de- } \\
\text { hospital }\end{array}$ \\
\hline 8 & $16 / 09 / 2017$ & $\begin{array}{l}\text { Hospital Luis } \\
\text { Negreiros (Callao) }\end{array}$ & $\begin{array}{l}\text { Frente al } \\
\text { hospital }\end{array}$ & 30 años & Ojo & $\begin{array}{l}\text { https://ojo.pe/ciudad/callao-mujer- } \\
\text { embarzada-da-a-luz-en-jugueria- } \\
\text { y-frente-al-hospital-negreiros- } \\
\text { video-246772/ }\end{array}$ \\
\hline
\end{tabular}




\section{DISCUSIÓN}

La saturación y disminución de la calidad de las intervenciones en los establecimientos de salud, las barreras culturales entre el profesional de salud y la gestante, la falta de respeto y el escaso ejercicio de los derechos sexuales y reproductivos ${ }^{3}$, entre otros factores, serían mediadores de esta problemática.

En los últimos 10 años, en Lima Metropolitana (principalmente en la zona norte) y el Callao el número de muertes maternas muestra tendencia al aumento ${ }^{4}$, en contraste a la mejora en la cobertura de partos institucionales, así como en la oportunidad y cantidad de controles pre-natales ${ }^{5}$. Es decir, la mejora en el acceso a esta intervención sanitaria podría estar condicionando una creciente saturación de los servicios hospitalarios -por la atención de partos normales- y la consiguiente disminución en la calidad dela atención delas urgencias obstétricas ${ }^{8,9}$ exacerbada por la inequidad y la falta de empoderamiento de las mujeres para ejercer sus derechos ${ }^{8}$; no se sienten protagonistas de sus partos, pues consideran que existen pautas preestablecidas en las que se tiene poco en cuenta su opinión o preferencias. Acuden al parto con expectativas muy superiores respecto a su autonomía y poder de decisión, pero no las encuentran en la realidad 6 .

En un reciente estudio realizado en el Hospital Sergio Bernales, Sevillano ${ }^{10}$ observó que $70,8 \%$ de los obstetras y ginecólogos realizaban prácticas inadecuadas relacionadas a la atención de parto humanizado. Este problema no sería reciente; Tarqui y Barreda11, hace más de diez años observaron que uno de los principales factores -asociados a la preferencia por el parto domiciliario- era el poco entendimiento entre proveedor y usuaria durante la atención de salud. Esto posiblemente se deba a que el personal sanitario asume un papel "funcional"11 sin tomar en consideración las creencias y costumbres de la mujer ${ }^{12}$, además de emplear un lenguaje poco comprensible durante la atención, lo que conlleva al poco entendimiento de las indicaciones médicas descrito por Mayca y col. ${ }^{13}$.

Como vemos, estos casos evidenciarían deficiencias en el acceso a la atención del parto de calidad y podrían ser la punta del iceberg de una inadecuada y deshumanizada atención integral de la salud materna, por lo que se requiere mayor estudio para identificar los determinantes estructurales e intermediarios que estarían mediándolo y así dirigir las intervenciones que eliminen este obstáculo que no permite lograr la salud materna en nuestro país.

Contribuciones de autoría: Los autores participaron en la generación, redacción y aprobación final del artículo original.

\section{Financiamiento: Autofinanciado.}

Conflicto de interés: Los autores declaran no tener conflicto de interés en la publicación de este artículo.

Recibido: 20 de agosto del 2018

Aprobado: 09 de noviembre del 2018

Correspondencia: Alfredo E. Oyola García

Dirección: Parque Leoncio Prado 145 -Dpto. 304, Magdalena del Mar, Lima-Perú Oficina: 51-56-224163/Celular: 51-956124996/RPM: *345700

Correo:aoyolag@gmail.com

\section{REFERENCIAS BIBLIOGRÁFICAS}

1. Suárez-Cortés M, Armero-Barranco D, Canteras-Jordana M, MartínezRoche ME. Uso e influencia de los planes de parto y nacimiento en el proceso de parto humanizado. Rev. Latino-Am. Enfermagem (internet). 2015 (citado 5 ene 2018); 23(3):520-526. Disponible en: http://www. scielo.br/pdf/rlae/v23n3/es_0104-1169-rlae-0067-2583.pdf

2. Ministerio de Salud. NTS N 105-MINSA/DGSP-V.01:"Norma Técnica de Salud para la Atención Integral de Salud Materna" (internet). Resolución Ministerial No 827-2013/MINSA (citado 5 ene 2018). Disponible en http://www.unfpa.org.pe/Legislacion/PDF/20131224-MINSA-NTAtencion-Salud-Materna.pdf

3.UNFPA Perú. Regiones con altas tasas de mortalidad materna enfrentan serios desafíos para asegurar la calidad en la atención de la salud materna (internet). 2017. (citado 5 ene 2018). Disponible en: http:// peru.unfpa.org/es/noticias/regiones-con-altas-tasas-de-mortalidadmaterna-enfrentan-serios-desaf\%C3\%ADos-para-asegurar-la

4. Centro Nacional de Epidemiología, Prevención y Control de Enfermedades. Sala situacional para el Análisis de Situación de Salud - SE 42-2017. 2017 Muerte materna (internet). (citado 5 ene 2018). Disponible en: http://www.dge.gob.pe/portal/index.php?option=com content\&view=article\&id=14\&ltemid $=121$
5. Instituto Nacional de Estadística e Informática. Perú: Indicadores de resultados de los programas presupuestales, 2011 - 2016. Encuesta demográfica y de salud familiar (resultados preliminares) (internet). Lima: Instituto Nacional de Estadística e Informática. 2017; pág. 129. (citado 5 ene 2018). Disponible en: https://proyectos.inei.gob.pe/ endes/ppr.asp

6. Molina Al, Román MR. Expectativas y experiencias de parto entre mujeres primíparas. Estudio cualitativo. Paraninfo Digital (internet). 2014 (citado 5 ene 2018); 8(20):1-7. Disponible en: http://www.index-f. com/para/n20/pdf/042.pdf

7. Montesinos-Segura R, Taype-Rondán A. ¿Qué sabemos sobre la falta de respeto y maltrato durante la atención del parto en el Perú? [carta]. Rev Peru Med Exp Salud Publica (internet). 2015 (citado 5 ene 2018); 32(3):608-610. Disponible en: https://scielosp.org/pdf/rpmesp/v32n3/ a32v32n3.pdf

8. Del Carpio L. Situación de la mortalidad materna en el Perú, 20002012. Rev Peru Med Exp Salud Publica (internet). 2013 (citado 5 ene 2018); 30(3):461-464. Disponible en: http://www.ins.gob.pe/insvirtual/ images/artrevista/pdf/rpmesp2013.v30.n3.a15.pdf 
9. Sánchez HJ, Arana M. La muerte materna en Chiapas: una historia inacabada. Medicina Social (internet). 2014 (citado 5 ene 2018); 9(1):42-44. Disponible en: http://www.medicinasocial.info/index.php/ medicinasocial/article/view/814

10.Sevillano M. Conocimientos, actitudes y prácticas relacionadas a la atención de parto humanizado en los profesionales del servicio de gineco-obstetricia del hospital nacional Sergio E. Bernales. Febreromarzo 2016 (internet). Lima: Universidad Nacional Mayor de San Marcos. 2016 (citado 5 ene 2018); pág. 59. Disponible en: http://cybertesis. unmsm.edu.pe/bitstream/cybertesis/4741/1/Sevillano $\mathrm{rm}$. pdf

11. Tarqui C, Barreda A. Factores asociados con la elección del parto domiciliario en una zona de atención primaria. Callao, Perú. Rev Peru Med Exp Salud Publica (internet). 2005 (citado 5 ene 2018); 22(2):109116. Disponible en: http://www.rpmesp.ins.gob.pe/index.php/rpmesp/ article/view/1016/1016
12. Pinedo A, Baffigo V. Adecuación cultural en la atención del parto. Ginecología y Obstetricia (internet). 2000 (citado 5 ene 2018); 46(3):262-265. Disponible en: http://repebis.upch.edu.pe/articulos/ ginecol\&obstet/v46n3/a12.pdf

13. Mayca J, Palacios-Flores E, Medina A, Velásquez JE, Castañeda D. Percepciones del personal de salud y la comunidad sobre la adecuación cultural de los servicios materno perinatales en zonas rurales andinas y amazónicas de la región Huánuco. Rev Peru Med Exp Salud Publica (internet). 2009 (citado 5 ene 2018); 26(2):145-60. Disponible en: http:// www.ins.gob.pe/insvirtual/images/artrevista/pdf/rpmesp2009.v26. n2.a4.pdf

Consulte la Versión Electrónica de la Revista:

Facultad de Medicina Humana Universidad Ricardo Palma

http://revistas.urp.edu.pe/index.php/RFMH<smiles>C1=[C+]2CCC2C1</smiles>

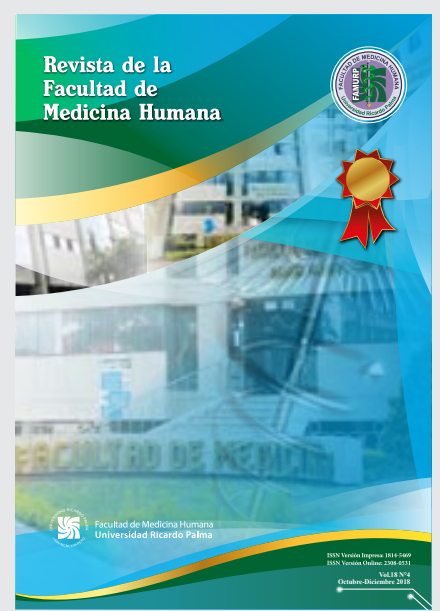

